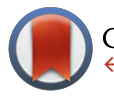

CrossMark

\& click for updates

Cite this: Lab Chip, 2016, 16, 1561

Received 7th December 2015,

Accepted 21st March 2016

DOI: $10.1039 / c 5 l c 01498 k$

www.rsc.org/loc

\section{Tacky cyclic olefin copolymer: a biocompatible bonding technique for the fabrication of microfluidic channels in COC}

\author{
Nico Keller, Tobias M. Nargang, Matthias Runck, Frederik Kotz, Andreas Striegel, \\ Kai Sachsenheimer, Denis Klemm, Kerstin Länge, Matthias Worgull, \\ Christiane Richter, Dorothea Helmer and Bastian E. Rapp*
}

Cyclic olefin copolymer (COC) is widely used in microfluidics due to its UV-transparency, its biocompatibility and high chemical resistance. Here we present a fast and cost-effective solvent bonding technique, which allows for the efficient bonding of protein-patterned $\mathrm{COC}$ structures. The bonding process is carried out at room temperature and takes less than three minutes. Enzyme activity is retained upon bonding and microstructure deformation does not occur.

\section{Introduction}

Cyclic olefin copolymer (COC) is a well suited material for microfluidic applications due to its numerous advantageous characteristics such as good biocompatibility, ${ }^{1}$ low water absorption properties, good chemical resistance ${ }^{2}$ and high transparency in the deep UV range. ${ }^{3} \mathrm{UV}$ transparent channel walls allow for the utilization of UV initiators such as benzophenone and its derivatives which are widely used for microfluidic western blotting ${ }^{4}$ and polymerization inside microfluidic channels, often to achieve varying surface properties by graft polymerization ${ }^{5}$ or polymerization of hydrogels. ${ }^{6}$ As a material for replication COC is used for the production of microfluidic chips ${ }^{7}$ for instance for lab-on-a-chip systems to analyse parameters like blood gas, glucose or lactate concentration $^{8}$ or for the determination of the haematocrit level. ${ }^{9}$

The immobilization of proteins inside microfluidic channels is becoming increasingly important for the production of microchannel enzyme reactors and microfluidic immunoassays. Numerous immobilization strategies for the immobilization of biomolecules inside microfluidic chips have been reported, see Kim and Herr for a review on the subject. ${ }^{10}$ For assay chips that detect several analytes at a time (multiplexed assays) or assays that require distinct reaction, washing and

Karlsruhe Institute of Technology (KIT), Institute of Microstructure Technology (IMT), Hermann-von-Helmholtz-Platz 1, 76344 Eggenstein-Leopoldshafen, Germany. E-mail: bastian.rapp@kit.edu; Fax: +49 (0)721 608 26667; Tel: +49 (0)721 60828981 mixing zones (multistep assays or reactors), the spatially directed immobilization of biomolecules or molecular patterns is essential. Microcontact printing $(\mu \mathrm{cP})^{11,12}$ and photoinitialized immobilization ${ }^{13}$ are the most common methods for generating protein patterns inside microfluidic channels. To facilitate washing steps it can be advantageous to seal the channel after photo-immobilization of the biomolecules on the inner channel surface. To seal microfluidic channels with a biomolecular-functionalized surface the bonding process needs to be executed under mild conditions to prevent biomolecule degradation. An ideal biocompatible bonding technique for biochemical applications thus has to ensure that the following criteria are met: (1) exposure of only one substrate to the solvent, i.e. the sealing lid without immobilized biomolecules on the surface, (2) prevention of protein degradation by applying only low temperatures during bonding (3) creation of bonds with high optical clarity to ensure the excellent optical properties of $\mathrm{COC}$ are retained e.g., for inchannel analysis or consecutive photo-polymerizations. Exposing only one substrate also avoids the problem of microstructure deformation upon bonding. ${ }^{15}$ Several COC bonding methods have been reported so far. The most important methods are thermal fusion bonding and solvent bonding. ${ }^{14}$ Thermal fusion bonding requires heating of the COC substrates above the $T_{\mathrm{g}}$ value, which causes immobilized biomolecules to denature and is therefore not generally a biocompatible method. Solvent bonding on the other hand combines the advantages of a low temperature process with high optical bond clarity. Several solvents have been reported for the use in solvent bonding of COC such as cyclohexane, ${ }^{15}$ methylcyclohexane, ${ }^{2}$ toluene ${ }^{16}$ anisole $/ n$-heptane ${ }^{16}$ and ethanol/decalin. ${ }^{17}$ Some techniques require the exposure of just the $\operatorname{lid}^{15,18}$ or both the lid and the channel ${ }^{19}$ structures to the solvent, some need special equipment like hydraulic presses $^{17,19}$ application of high process pressures or additional heating during pressing. ${ }^{2,17,18,20}$ Although high process pressure and high process temperature increase the strength of COC/COC bonds ${ }^{7}$ they cannot be used if ligands such as 
enzymes or antibodies have previously been immobilized on the substrates.

In this paper we present a COC solvent bonding technique that allows for biocompatible room temperature bonding of protein-patterned COC, requires no additional equipment and produces bond strength well above $744 \mathrm{kPa}$. We show that our method is compatible with biomolecules such as proteins immobilized on the COC substrates. The method relies on a 3 min solvent immersion and subsequent drying process which turns the surface of the COC tacky. Such "tacky COC" lids can be used as a biocompatible bonding technique for microfluidic chips made in COC.

\section{Results}

We used TOPAS 8007S-04 COC films with a thickness of $130 \mu \mathrm{m}$ for bonding experiments. Our liquid solvent bonding technique is based on the following steps (see Fig. 1): the COC lid is cleaned with 2-propanol and dried with compressed air. For the coating step we placed three layers of filter paper (Carl Schleicher \& Schüll no. 589/3) in a glass Petri dish. The filter paper is soaked with the desired mixture of solvents. In the "coating" step, the COC lid is placed on the soaked paper for $1 \mathrm{~min}$. After coating the COC film is cleaned using acetone and dried with compressed air turning the surface tacky. The tacky lid is then pressed onto the COC substrate with a force of $90 \mathrm{~N}$ for $30 \mathrm{~s}$. It is possible to use the tacky lid for up to $10 \mathrm{~min}$ after drying.

COC dissolves preferably in non-polar organic solvents such as hydrocarbons, while it is resistant to polar organic solvents like acetone. ${ }^{21}$ Hydrocarbons like cyclohexane readily penetrate into the COC polymer, causing it to swell by spacing the polymeric chains thus turning the surface "tacky" until all solvent has evaporated. In the tacky state COC substrates can be pressed together to form a stable bond due to residual solvent penetrating into the non-tacky COC surface and jamming of the solvent-spaced polymer chains. To determine the optimum bonding conditions, we first characterized the influence of the solvent mixture on the transparency of the bond. For this test we bonded two pieces of COC film $(2 \mathrm{~cm} \times 2 \mathrm{~cm})$ with different mixtures of cyclohexane, toluene and $n$-hexane. We measured the UV/Vis transmittance of the bonded substrates, some of which are shown in Fig. 2. Neat hydrocarbons such as cyclohexane penetrate deeply into the

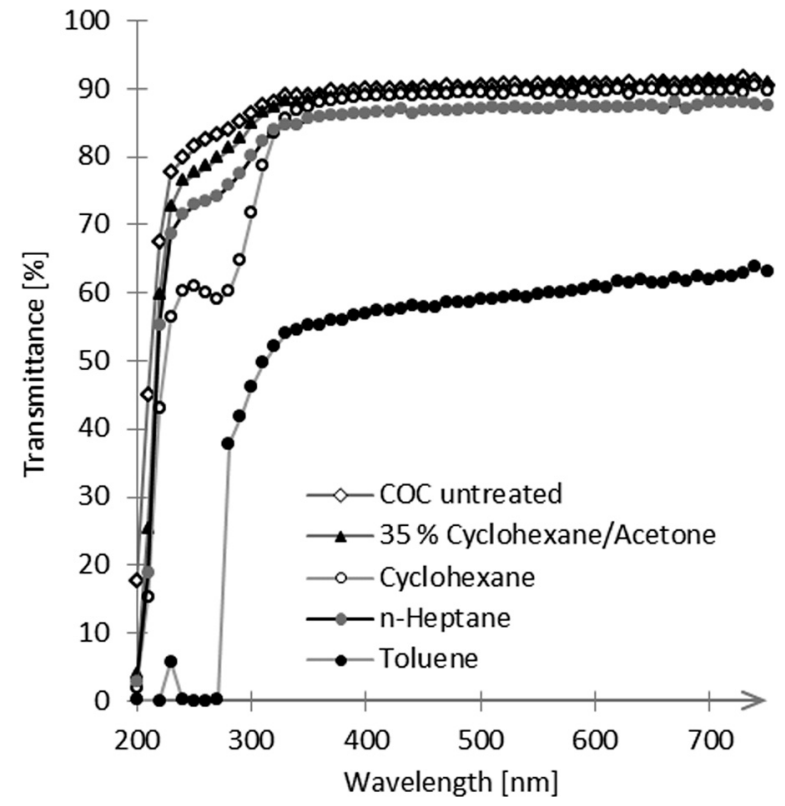

Fig. 2 Optical transmission of $\mathrm{COC}$ substrates before ( $\mathrm{COC}$ untreated) and after bonding with different solvents or solvent mixtures.

COC network. Over time the solvent is released causing the formation of bubbles at the bonding interface (see Fig. 3).

Dilution of hydrocarbons with a non-penetrating polar solvent prevents the excessive absorption of solvent thus leading to less or no bubble formation. The bond with cyclohexane showed the best optical transmission at $405 \mathrm{~nm}$, a wavelength especially relevant for the detection of enzyme activity

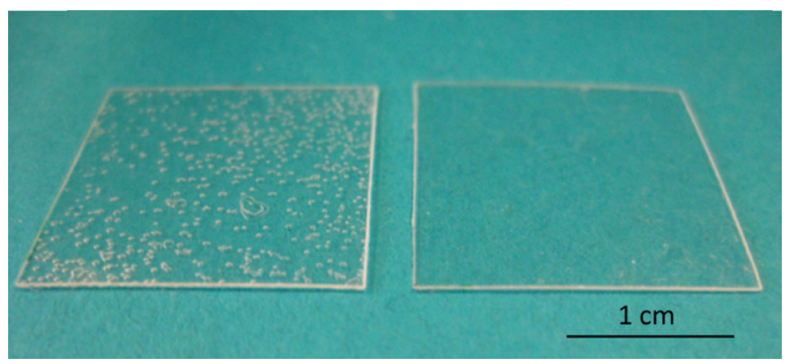

Fig. 3 Influence of the solvent on solvent bonding results. With pure solvents (left: cyclohexane) the formation of bubbles is observed, whilst optical clarity can be achieved by bonding with appropriate solvent mixtures (right: $35 \%$ cyclohexane in acetone). a)

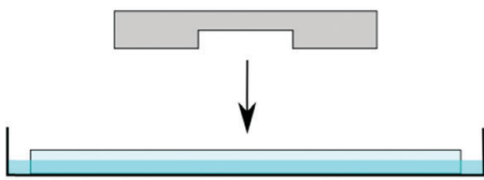

b)

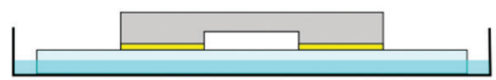

Tacky COC solvent

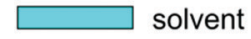

c)

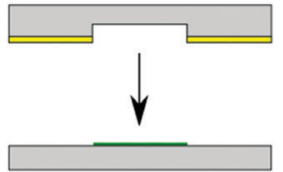

d)

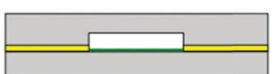

$\mathrm{COC}$

filter paper

functionalized layer

Fig. 1 Bonding of a COC lid to a bio-functionalized COC substrate using the "tacky COC" technique. (a-b) Rendering the COC surface tacky by placing the $\mathrm{COC}$ film on a cyclohexane/acetone soaked filter paper for $1 \mathrm{~min}$. (c-d) Bonding of tacky COC onto a COC lid with immobilized proteins on the surface by pressing the tacky $\mathrm{COC}$ onto the functionalized substrate for $30 \mathrm{~s}$. 
in an horseradish peroxidase (HRP) enzyme assay. Therefore we decided to dilute cyclohexane (hydrocarbon) with acetone (polar solvent) to reduce the solubility of COC and prevent bubble formation between the COC sheets while maintaining high optical clarity. For a summary of the achieved bonds and their characterization see Table 1. We found $35 \mathrm{vol} \%$ cyclohexane in acetone to give the best results with transmissions of $77 \%$ at $250 \mathrm{~nm}$ compared to $80 \%$ in untreated COC and transmission of $89 \%$ at $405 \mathrm{~nm}$ compared to $90 \%$ in untreated COC (see Fig. 2).

The bond strength of the 35 vol\% cyclohexane/acetone bond was characterised with a burst pressure test. COC film $(1 \mathrm{~cm} \times 1 \mathrm{~cm})$ was bonded onto a $3 \mathrm{~mm}$ COC plate with a hole for the compressed air line connection (see Fig. 4). After overnight storage at room temperature, air of increasing pressure was applied to the bonded sheets. At a maximum pressure of $744 \mathrm{kPa}$ the COC/COC bond showed no leakage which is sufficient for all standard microfluidic applications.

For the immobilization of biomolecules on COC we created patterns of biotin using a lithographic method that has been previously described. ${ }^{22}$ Briefly, the COC substrates were coated with BSA (bovine serum albumin) by immersion in 3 wt $\%$ BSA in PBS (phosphate buffered saline), washed and dried in a stream of nitrogen. Photo-immobilization of biotin was achieved by applying $80 \mu \mathrm{M}$ fluorescein-5-biotin (F5B) in PBS onto the BSA coating and exposing the surface to light (490 nm). This induced radical formation due to photobleaching of fluorescein thus resulting in a covalent attachment of F5B on the BSA layer. As opposed to $\mu \mathrm{cP}$ photoimmobilization of biomolecules in microfluidic channels

Table 1 Overview of tacky COC bonding tests with different cyclohexane/acetone mixtures

\begin{tabular}{lllll}
\hline $\begin{array}{l}\text { Vol\% of } \\
\text { cyclohexane } \\
\text { in acetone }\end{array}$ & Bond & $\begin{array}{l}\text { Optical } \\
\text { properties }\end{array}$ & \multicolumn{2}{l}{ Transmittance [\%] } \\
\cline { 4 - 5 } 10 & $\mathrm{No}^{a}$ & Clear & $-^{c}$ & $--^{c}$ \\
20 & $\mathrm{No}^{b}$ & Clear & $-^{c}$ & $-^{c}$ \\
35 & Yes & Clear & 77 & 89 \\
50 & Yes & Bubble formation & 68 & 84 \\
70 & Yes & Bubble formation & 64 & 83 \\
100 & Yes & Bubble formation & 61 & 89
\end{tabular}

${ }^{a}$ No tacky layer is formed. It was not possible to bond the tacky COC with another COC sheet. ${ }^{b} \mathrm{~A}$ tacky layer is formed and bonding is possible. However, the bond formed was weak and the substrates could easily be removed with tweezers. ${ }^{c}$ Because no stable bond had formed, no transmittance measurement was carried out.

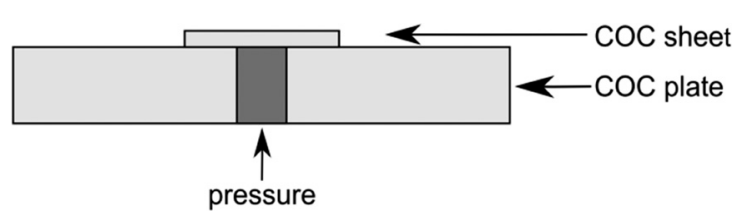

Fig. 4 Schematic of the burst pressure test. The COC sheet $(1 \mathrm{~cm} \times 1 \mathrm{~cm})$ has a thickness of $130 \mu \mathrm{m}$ and the COC plate has a thickness of $3 \mathrm{~mm}$. can be executed inside already sealed channels. We found however, that washing steps are greatly facilitated in open channel structures leading to biotin patterns of significantly enhanced quality (see Fig. 5). Combined with a biocompatible bonding method, we found this approach superior to exposure and washing inside sealed channels.

To prove the biocompatibility of our bonding technique, we immobilized streptavidin-labelled horseradish peroxidase (R\&D Systems) by incubating a biotin-covered channel with 1:200 dilute enzyme solution in PBS for $60 \mathrm{~min}$. The channel was rinsed with deionised water, washed three times in $10 \mathrm{~mL}$ PBS (for HRP) for $5 \mathrm{~min}$ each and once with deionised water and dried under a flow of nitrogen. Channels were sealed by the tacky COC method and the activities of the HRP inside the channels was tested by using an enzyme substrate that produce colours upon conversion: the channel was incubated with $10 \mu \mathrm{L}$ 2,2'-azino-bis(3-ethylbenz-thiazoline-6sulphonic acid) (ABTS) reaction solution consisting of $1 \mathrm{ml}$ of $1 \mathrm{mg} \mathrm{mL}^{-1}$ ABTS in acetate buffer $(25 \mathrm{mM}$ acetic acid, $4.45 \mathrm{mM}$ sodium acetate $\mathrm{pH}$ 4.0) mixed with $100 \mu \mathrm{L} 0.01 \%$ hydrogen peroxide. After $60 \mathrm{~min}$ a green colour had developed inside the channel indicating an active enzyme (see Fig. 6 inset). For comparison equivalently coated HRP channels were sealed by thermal fusion bonding at $103{ }^{\circ} \mathrm{C}$ for 30 min with a force of $18.6 \mathrm{~N}$ and incubated with ABTS reaction solution. Substrate conversion in channels bonded by the tacky COC method and those bonded by thermal fusion were quantified by measuring the absorption at $405 \mathrm{~nm}$ (see Fig. 6). A significantly lower amount of substrate was processed inside the channels bonded by thermal fusion indicating an inactive enzyme due to heat denaturation.

To ensure a wider applicability of the method, we also tested for microstructure deformation upon bonding. We bonded a tacky wall and lid structure onto a hot embossed COC microstructure. After removal of the lid REM images of the microstructure were taken. As shown in Fig. 7 the structures were not affected by the bonding process making it applicable for structured as well as patterned channel surfaces.
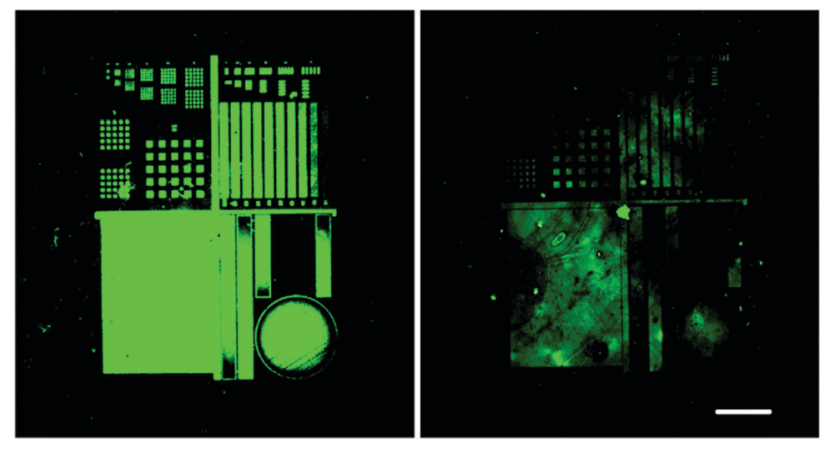

Fig. 5 Biotin pattern inside channels sealed by tacky COC bonding visualized by streptavidin-Cy3. Left: Channels sealed after photoimmobilization and staining. Right: Channel sealed before photoimmobilization and washing. Washing and staining are significantly facilitated in open channel structures and lead to higher quality protein patterns. Scale bar: $500 \mu \mathrm{m}$, total bitmap size: $2.5 \mathrm{~mm} \times 1.92 \mathrm{~mm}$. 


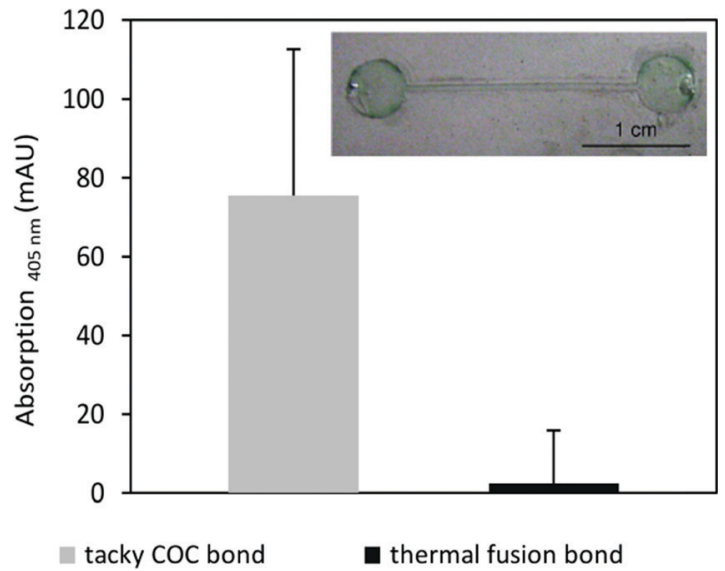

Fig. 6 Quantification of ABTS conversion by HRP immobilized in microfluidic channels. Substrate conversion was detected by absorption measurements at $405 \mathrm{~nm}$ inside a channel sealed by the tacky $\mathrm{COC}$ method and one sealed by thermal fusion. Only during bonding of tacky COC lids enzyme activity is retained. Inset: HRP coated channel sealed by the tacky COC method after incubation with ABTS enzyme substrate solution.

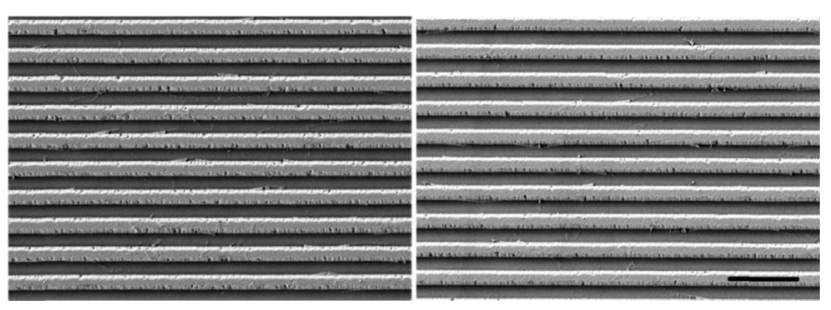

Fig. 7 COC microstructure (made by hot embossing) before (left) and after (right) bonding of a tacky wall and lid structure. Microstructures were not deformed by tacky COC bonding. Scale bar: $4 \mu \mathrm{m}$.

\section{Conclusion}

The tacky COC bonding method is a fast and simple bonding technique for COC and unlike other state-of-the-art techniques requires no additional equipment like hot plates or hydraulic presses, high temperature or UV exposure steps. The bonded substrates possess optical clarity close to untreated COC. The bonding method is biocompatible and can be used to seal sensitive substrates such as proteincoated COC channels without the loss of enzyme activity. Due to the exposure of only the channel lid structures microstructure deformation is not an issue.

\section{Acknowledgements}

The authors thank P. Abaffy for providing the REM images. This work was funded by the German Ministry of Education and Research (BMBF), funding code 03X5527 "Fluoropor".

\section{References}

1 P. S. Nunes, P. D. Ohlsson, O. Ordeig and J. P. Kutter, Microfluid. Nanofluid., 2010, 9, 145-161.

2 K. W. Ro, J. Liu and D. R. Knapp, J. Chromatogr. A, 2006, 1111, 40-47.

3 A. Piruska, I. Nikcevic, S. H. Lee, C. Ahn, W. R. Heineman, P. A. Limbach and C. J. Seliskar, Lab Chip, 2005, 5, 1348-1354.

4 A. J. Hughes and A. E. Herr, Proc. Natl. Acad. Sci. U. S. A., 2012, 109, 21450-21455.

5 S. W. Hu, X. Q. Ren, M. Bachman, C. E. Sims, G. P. Li and N. L. Allbritton, Anal. Chem., 2004, 76, 1865-1870.

6 D. J. Beebe, J. S. Moore, J. M. Bauer, Q. Yu, R. H. Liu, C. Devadoss and B. H. Jo, Nature, 2000, 404, 588-590.

7 J. Steigert, S. Haeberle, T. Brenner, C. Müller, C. Steinert, P. Koltay, N. Gottschlich, H. Reinecke, J. Rühe and R. Zengerle, J. Micromech. Microeng., 2007, 17, 333.

8 C. H. Ahn, J.-W. Choi, G. Beaucage, J. H. Nevin, J.-B. Lee, A. Puntambekar and J. Y. Lee, Proc. IEEE, 2004, 92, 154-173.

9 L. Riegger, M. Grumann, J. Steigert, S. Lutz, C. Steinert, C. Mueller, J. Viertel, O. Prucker, J. Rühe and R. Zengerle, Biomed. Microdevices, 2007, 9, 795-799.

10 D. Kim and A. E. Herr, Biomicrofluidics, 2013, 7, 401501.

11 A. Kumar and G. M. Whitesides, Appl. Phys. Lett., 1993, 63, 2002-2004.

12 A. Khademhosseini, K. Y. Suh, S. Jon, G. Eng, J. Yeh, G. J. Chen and R. Langer, Anal. Chem., 2004, 76, 3675-3681.

13 M. A. Holden, S. Y. Jung and P. S. Cremer, Anal. Chem., 2004, 76, 1838-1843.

14 C.-W. Tsao and D. L. DeVoe, Microfluid. Nanofluid., 2009, 6, 1-16.

15 D. A. Mair, M. Rolandi, M. Snauko, R. Noroski, F. Svec and J. M. Fréchet, Anal. Chem., 2007, 79, 5097-5102.

16 S. Herrlich, T. Lorenz, S. Spieth, S. Messner and R. Zengerle, Solvent Bonding of a Drug Delivery Device by Using Hansen Solubility Parameters, 2011.

17 T. I. Wallow, A. M. Morales, B. A. Simmons, M. C. Hunter, K. L. Krafcik, L. A. Domeier, S. M. Sickafoose, K. D. Patel and A. Gardea, Lab Chip, 2007, 7, 1825-1831.

18 K. Faure, M. Albert, V. Dugas, G. Crétier, R. Ferrigno, P. Morin and J. L. Rocca, Electrophoresis, 2008, 29, 4948-4955.

19 C. Chen, J. Liu, L. Hromada, C. Tsao, C. Chang and D. DeVoe, Lab Chip, 2009, 9, 50-55.

20 I. Ogilvie, V. Sieben, C. Floquet, R. Zmijan, M. Mowlem and H. Morgan, J. Micromech. Microeng., 2010, 20, 065016.

21 C. M. Hansen and L. Just, Ind. Eng. Chem. Res., 2001, 40, 21-25.

22 A. Waldbaur, B. Waterkotte, K. Schmitz and B. E. Rapp, Small, 2012, 8, 1570-1578. 